\title{
Handbuch Forschungsdatenmanagement
}

Herausgegeben von Stephan Büttner, Hans-Christoph Hobohm, Lars Müller

BOCK + HERCHEN Verlag Bad Honnef 
Die Inhalte dieses Buches stehen auch als Online-Version zur Verfügung: www.forschungsdatenmanagement.de

Die Onlineversion steht unter folgender Creative-Common-Lizenz: „Attribution-NonCommercial-ShareAlike 3.0 Unported“ http://creativecommons.org/licenses/by-nc-sa/3.0/

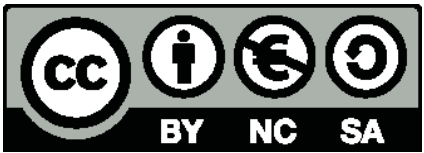

ISBN 978-3-88347-283-6

BOCK+HERCHEN Verlag, Bad Honnef

Printed in Germany 


\subsection{Datenanalyse und -visualisierung}

Bettina Berendt [1], Joaquin Vanschoren [2], Bo Gao [1]

[1] K.U. Leuven, Belgien

[2] Universiteit Leiden, Niederlande

Das Management von Forschungsdaten wird dann interessant, wenn die Datenhaltung nicht nur eine Dokumentationsfunktion erfüllt, sondern es erlaubt, Daten in neuen Weisen wieder- und weiterzunutzen. Repositorien für das Forschungsdatenmanagement (FDM) stellen daher idealerweise nicht nur Mechanismen für das Speichern und Finden von Daten zur Verfügung, sondern auch die Grundlagen oder auch die Tools für Analysen auf diesen Daten, die über die schon im „Ursprungsprojekt“ der Daten geleisteten hinausgehen.

Visualisierungen erscheinen im FDM in zweierlei Form: Zum einen können Visualisierungen Objekte, Materialien oder Ergebnisse der dokumentierten Forschung sein - beispielsweise kann eine medien-, kunst- oder sozialwissenschaftliche Datenbank Fotografien oder Filme als Studienobjekt enthalten, als in einer empirischen Untersuchung eingesetzter Stimulus, oder als Dokumentation einer Interviewreihe. Zum anderen können Visualisierungen Methode der dokumentierten Forschung sein - oder auch Methode der Forschung, die erst durch FDM möglich wird.

Eine typische Methode der dokumentierten Forschung sind etwa Datengraphiken, die vorrangig der Präsentation von Messdaten oder Informationen dienen. Visualisierungen können aber darüber hinaus auch der Exploration dienen. Erst durch FDM möglich wird die Exploration der Gesamtheit der verwalteten Daten. Visuelle wie nicht-visuelle explorative Datenanalysen spielen eine wesentliche Rolle in der Generierung neuer Forschungsideen und Hypothesen (z. B. Tukey, 1977; Hand, Smyth \& Mannila, 2001) und sind damit eine der Kernmotivationen, FDM zu betreiben. ${ }^{1}$ Wir fokussieren daher auf Datenvisualisierung als eine Form der Datenanalyse und sprechen allgemein von Analyse/ Visualisierung.

Die Frage, was Analyse/Visualisierung für das Forschungsdatenmanagement ist, lässt sich nun in zweierlei Weise konkretisieren: einerseits, was alles Analyse/Visualisierung für das FDM sein kann; andererseits, was Analyse/Visualisierung speziell für das FDM ist. Daher muss weiter gefragt werden, (a) was Forschungsdaten sind, (b) welche Daten speziell in Forschungsdaten-Datenbanken auftauchen, (c) welche Anforderungen das FDM-Ziel „Analyse“ an Daten und Visualisierungsformen stellt.

1. Natürlich können auch Datenanalysen Objekt, Material oder Methode der dokumentierten Forschung sein. 


\subsubsection{Welche Anforderungen stellt die FDM-Aufgabe der (u.a. visuellen) Datenanalyse an Daten und an Visuali- sierungsformen?}

Zur Beantwortung der Frage nach den Anforderungen führen wir zunächst den Begriff des FDM auf den allgemeineren des Datenmanagement zurück: „das Datenmanagement ist die Menge aller methodischen, konzeptionellen, organisatorischen und technischen Maßnahmen und Verfahren zur Behandlung von ,Daten' [mit dem Ziel] der Gewährleistung einer maximalen Unterstützung auszuführender Geschäftsprozesse ...“ (Schenk, 2010, S. 316). Diese allgemeine Definition muss instanziiert werden: Wir betrachten als die zu unterstützenden „Geschäftsprozesse“ verschiedenste Prozesse der eScience und betrachten in diesem Artikel speziell Fragen zu methodischen, organisatorischen und technischen Aspekten einer hierfür geeigneten Infrastruktur und dabei besonders Methoden zur Auswertung der Daten mit dem Ziel der Wissensgewinnung sowie Aspekte der technischen (Implementation) und organisatorischen (Web-Basierung) Realisierung dieser Methoden. Schließlich sind die ,Daten' des Forschungsdatenmanagements natürlich Forschungsdaten. Forschungsdaten umfassen u.a. (i) Dokumente, z. B. Textdokumente oder Tabellenkalkulationsdokumente, (ii) Laborbücher, Feldaufzeichnungen, weitere Logbücher, (iii) Fragebögen, Transkripte, Codebücher, (iv) Audio- und Videoaufzeichnungen, (v) Fotografien und Filme, (vi) Testantworten, (vii) Dias, Artefakte, Muster, Proben, (viii) Sammlungen digitaler Objekte, die während des Forschungsprozesses angelegt wurden, (ix) Daten-Dateien, (x) Datenbankinhalte verschiedener Formate, (xi) Bestandteile einer Anwendung (Input, Output, Logfiles für die Analyse-Software, Simulationssoftware, Schemata), (xii) Methodologien und Workflows, und (xiii) Standardvorgehensweisen und Protokolle. Des Weiteren kann es sinnvoll sein, Daten wie z. B. Korrespondenz oder Projektfinanzierungsanträge über den konkreten Forschungszusammenhang, in dem sie entstanden sind, aufzubewahren (University of Edinburgh Information Services, 2009).

FDM kann dann einen Mehrwert erzeugen, wenn solche Daten nicht nur existieren, sondern auch zugreifbar, auffindbar/recherchierbar und (meta-)analysierbar sind. Hieraus ergeben sich Anforderungen für Infrastruktur und Methoden inkl. möglicher Visualisierungen, die jedoch für die meisten der oben genannten Datenformen nicht spezifisch für das FDM sind. So stellen beispielsweise Dokumente Anforderungen an eine FDM-Datenbank, die kaum von denen normaler Websuchmaschinen abweichen: Datenzugriff kann beispielsweise durch die Zuweisung persistenter URLs an die Dokumente und Verwendung von HTTP gewährleistet werden, Information-Retrieval-Methoden ermöglichen Auffindbarkeit und Recherchierbarkeit, und Standardverfahren z. B. des Text Mining und Visual Text Mining (vgl. Feldman \& Sanger, 2007) können wahlweise auf einem Server oder lokal von einem Client-Tool angeboten werden, um die Dokumente zu analysieren. (Handelt es sich nicht oder nicht vor- 
wiegend um Textdokumente, so können weitere Verfahren z. B. zum Visual Information Retrieval und Multimedia-Mining (Enser, 2008) zur Anwendung kommen.) Somit kann diese Beobachtung mindestens auf FDM-Daten der Formen (i), (ii), (iii), (iv), (v), (vi), (viii) und (xiii) verallgemeinert werden.

Auch weitere Formen von Forschungsdaten können von Standardtechnologie profitieren: Sollen Inhalte von (je nach am FDM beteiligten Partner wahrscheinlich unterschiedlicher) Datenbanken zugänglich gemacht werden, so eignen sich Techniken für Föderierte Datenbanken (z. B. Conrad, 1997) bzw. für das (Semantic) Web. Auch Dokumente können Inhalte in diesem Sinn sein. Daten als solche (ob in Datei- oder Datenbankform) können von verschiedenen Formen der Informationsvisualisierung profitieren (z. B. Schumann \& Müller, 1999). Für einige Formen von FDM-Daten wie z. B. Workflows gibt es Standards für interoperable Notation und auch für die Visualisierung einzelner Instanzen sowie Meta-Analysen.

Was also ist anders im FDM? Wir wollen hier die Grundidee der eSciences verfolgen: der Wissenschaften mit hohen Anforderungen an Rechenleistung, die das Internet als einen globalen, kollaborativen Arbeitsplatz nutzen, s. z. B. (Nielsen, 2008). Hierbei nehmen die Verwaltung und (Nach-)Nutzung von Daten (z. B. Messdaten) sowie Bestandteilen einer Anwendung (Input, Output, Prozessdaten) eine wichtige Rolle ein. Somit können z. B. Beobachtungs- oder Experimentaldaten mit hoher Detailtiefe notiert, ausgetauscht und kollaborativ vervollständigt werden, da viel einfacher und/oder systematischer als beispielsweise bei einer Literaturstudie sichtbar wird, welche Parameterkombinationen eines bestimmten Experimentallaufs untersucht worden sind und mit welchem Ergebnis, und welche noch nicht untersucht worden sind. ${ }^{2}$

\subsubsection{Experimentaldatenbanken als ein typisches Element von eScience und FDM}

Es ist daher nicht erstaunlich, dass in allen eSciences Online-Infrastrukturen gebaut werden, die dem Austausch von Experimenten dienen. Solche Infrastruk-

2. Selbstverständlich gilt für jegliche Offenlegung wissenschaftlicher Daten - genau wie für Daten anderer Datenproduzenten oder -halter auch - dass diese u.U. Privacy relevant sein können. Es muss daher in jedem Einzelfall sorgfältig geprüft werden, ob datenschutzrechtliche Fragen hinreichend berücksichtigt sind. Hierbei ist auch zu beachten, dass ,,anonymisierte“ Daten derzeit zwar nicht oder nur vermindert unter das Datenschutzrecht fallen, dass solche Anonymität aber in vielen Fällen durch - häufig einfache - Analyseoperationen hinfällig wird und somit de facto schwerwiegende Verletzungen der Privatsphäre möglich werden (z. B. Sweeney, 2002; Barbaro \& Zeller, 2006). Das Forschungsgebiet des Privacy-preserving Data Publishing analysiert derartige Probleme und Lösungsansätze; für einen Überblick, s. Fung et al., 2010). Zu juristischen Fragen des FDM s.a. Spindler und Hillegeist in diesem Band. 
turen beruhen auf drei wesentlichen Komponenten: (a) einer formale Beschreibungssprache, (b) Ontologien zur Gewährleistung gemeinsamer Semantik und (c) einem durchsuchbaren Repositorium. Visualisierungen der Ontologien können das Verständnis der Ontologien befördern (s. Neher \& Ritschel, in diesem Band, zu Ontologien und Katifori et al., 2007, zu ihrer Visualisierung), und in Anfragen an das Repositorium können visuelle Anfragesprachen genutzt werden. Auch hier unterscheidet sich das FDM nicht von anderen Anwendungsgebieten der Ontologie-Visualisierung oder der visuellen Anfragesprachen.

Entscheidenden Mehrwert können Repositorien und Visualisierungen im FDM dann schaffen, wenn sie eine Zusammenschau und Verdichtung von Daten aus unterschiedlichen Quellen bieten, aus der neue Beobachtungen dieser Daten und Ideen oder Hypothesen für Anschlussuntersuchungen gewonnen werden können. Die Zusammenschau wird durch interoperable Syntax und Semantik, realisiert in den Ontologien/Schemata des Repositoriums, möglich gemacht. Es können dann beispielsweise statistische (Meta-)Analysen auf der Kombination von Datensätzen aus unterschiedlichen Messreihen unterschiedlicher Forschungsprojekte durchgeführt werden; oder das Data-Mining-Tool eines FDMPartners kann die Daten eines anderen FDM-Partners direkt laden und auswerten. Die Generierung einer Datenvisualisierung unterscheidet sich hierin vom Gesichtspunkt der Software her nicht von anderen Arten der Analyse wie beispielsweise einer statistischen Auswertung. Gerade für explorative Analysen, die immer nur teilautomatisiert sein können und daher auf menschliche Wahrnehmung und Verarbeitung angewiesen sind, können Visualisierungen jedoch oft besser verdichten als numerische oder textuelle Darstellungsformen.

Ein wesentliches Charakteristikum typischer Forschungsdaten sind ihre großen Mengen und eine oft hohe Dimensionalität der einzelnen Datensätze. Damit kommt Techniken des Data Mining (durch seinen Fokus auf Skalierbarkeit von Analysen) und Techniken der Dimensionalitätsreduktion ${ }^{3}$ (durch ihren Fokus auf Darstellbarkeit in 2D/3D/4D-Medien ${ }^{4}$ ) eine entscheidende Rolle zu. Anders ausgedrückt: Methoden zur Visualisierung hochdimensionaler Daten haben verschiedene Anwendungsgebiete, sind aber im FDM zum Zwecke der Informationsstrukturierung, -filterung und -kondensierung noch unverzichtbarer als

3. Beispiele sind PCA, Classical Scaling, Isomaps, LLE, Laplacian Eigenmaps, Diffusion Maps, Kernel PCA und Sammon Maps (s. Gao, 2010).

4. $3 \mathrm{D}$ ist heute noch überwiegend 2.5D auf 2D-Ausgabegeräten wie z. B.

Standardbildschirmen; eine weitere Dimension und damit bis zu 4D lassen sich durch Animation visualisieren. Aktuelle Entwicklungen wie z. B. stereoskopische Verfahren (,3D-Kino“) können die Dimensionalität der Medien erhöhen. Unabhängig von der Dimensionalität des Mediums können Visualisierungsformate auch höherdimensional sein. So können z. B. Parallelkoordinaten-Darstellungen beliebig viele Dimensionen auf einem 2D-Medium darstellen. 
anderswo, wobei regelmäßig Daten und Metadaten über Inputs, Outputs und prozedurale Elemente kombiniert werden müssen.

Wir wollen diese allgemeinen Beobachtungen an einem konkreten Beispiel zeigen und hierbei auch illustrieren, welche Design-Entscheidungen die Wahl einer geeigneten Visualisierung bestimmen können.

Die Datenbank enthält Experimente zum maschinellen Lernen, einem Teilgebiet der Informatik, in der die Entwicklung von Experimentaldatenbanken noch nicht so weit fortgeschritten ist wie in anderen naturwissenschaftlichen Disziplinen etwa der Bioinformatik, der Astronomie oder der Physik (Vanschoren et al., im Druck). Das Beispiel ist jedoch hinsichtlich der Frage des aktuellen Artikels besonders interessant, da hier Datenanalysetechniken nicht nur Gegenstand des FDM, sondern auch Gegenstand der dokumentierten Forschung sind. Aus Platzgründen werden wir uns auf zwei der in diesem Abschnitt als zentral identifizierten Elemente konzentrieren: Beschreibung der Daten in Form einer Ontologie und Visualisierung auf Basis von Dimensionalitätsreduktion.

\subsubsection{Fallbeispiel: Eine Experimentaldatenbank für maschi- nelles Lernen / Data Mining}

Das maschinelle Lernen beschäftigt sich mit der Induktion von beschreibenden und vorhersagenden Modellen von Daten. Diese Induktion ist ein wichtiger Bestandteil des Prozesses des Data Mining, der Wissensentdeckung in Daten. Eine typische Aufgabe im maschinellen Lernen ist das Klassifikationslernen. Hier ist eine große Zahl von Dateninstanzen gegeben, die mit Hilfe von Features (Merkmalen) beschrieben werden und von denen man für einige weiß, zu welcher Klasse sie gehören - dieses aber auch für die anderen vorhersagen will. Beispielsweise kann ein Mailprovider oder eine Suchmaschine das Ziel haben, Mails oder Webseiten als Spam (oder Nicht-Spam) zu klassifizieren. Dieses Klassenlabel kann für bestimmte Datenmengen gegeben sein (beispielsweise weil Nutzer frühere Mails als Spam gekennzeichnet haben); interessant ist natürlich die Vorhersage für neue, noch unbekannte und unklassifizierte Dokumente. Als Features eignen sich hier u.a. Textmerkmale (z. B. die verwendeten Wörter), Metadaten (z. B. die URL) und weitere automatisch extrahierbare Eigenschaften (z. B. die Verlinkung zu anderen Inhalten). Ein Klassifikationslern-Verfahren (z. B. Entscheidungsbaumlernen) kann verschiedene Parameter haben (z. B. wie „korrekt“ ein Blatt des Entscheidungsbaums klassifizieren muss). Ein parametrisiertes Verfahren hat auf einer gegebenen Datenmenge eine bestimmte Performanz (z. B. die Genauigkeit: wie viele Datensätze korrekt klassifiziert wurden). Auch die Datenmengen selbst haben Eigenschaften, beispielsweise wie die Klassen in ihnen verteilt sind. (Dieser Parameter ist wichtig, weil z. B. auf einer Datenmenge, die zu 99\% aus Nicht-Spam besteht, sogar ein primitives Verfahren, das alle Mails als Nicht-Spam klassifiziert, bis zu 99\% korrekt sein kann.) Die voll- 
ständige Beschreibung eines Experiments kann weitere Features beinhalten (z. B. zur Beschreibung des Testverfahrens, mit dessen Hilfe die Genauigkeit bestimmt wurde). Die derzeit größte Datenbank zur Beschreibung solcher Experimente im maschinellen Lernen inklusive der Ontologie, die ihrem Schema zugrunde liegt, ist unter http://expdb.cs.kuleuven.be zu finden; sie enthält Datensätze über mehr als 650,000 Experimente (Vanschoren et al., im Druck).

Die Ontologie wird in Form der Experiment Markup Language ExpML notiert und mit Instanzen gefüllt und in der Datenbank ExpDB gespeichert. Sie enthält die Konzepte und Strukturen des Data-Mining-Experimentalprozesses sowie die dabei verwendeten Algorithmen und Datenmengen und ihre Details. Wie andere Ontologien dient sie einerseits der Lesbarkeit sowohl durch Menschen als auch durch Software-Agenten. Im Geiste des Ontology-Engineerings wurden soweit wie möglich Konzepte und Relationen aus verwandten und erprobten Ontologien wiederverwendet, insbesondere die Top-Level-Klassen und -Relationen einschlägiger Ontologien aus der Bioinformatik und dem Data Mining (u.a. BFO, OBI, IAO sowie OntoDM und DMOP; für Details s. Vanschoren et al., im Druck). Abbildung 1 zeigt eine (manuell erstellte) Visualisierung der Ontologie.

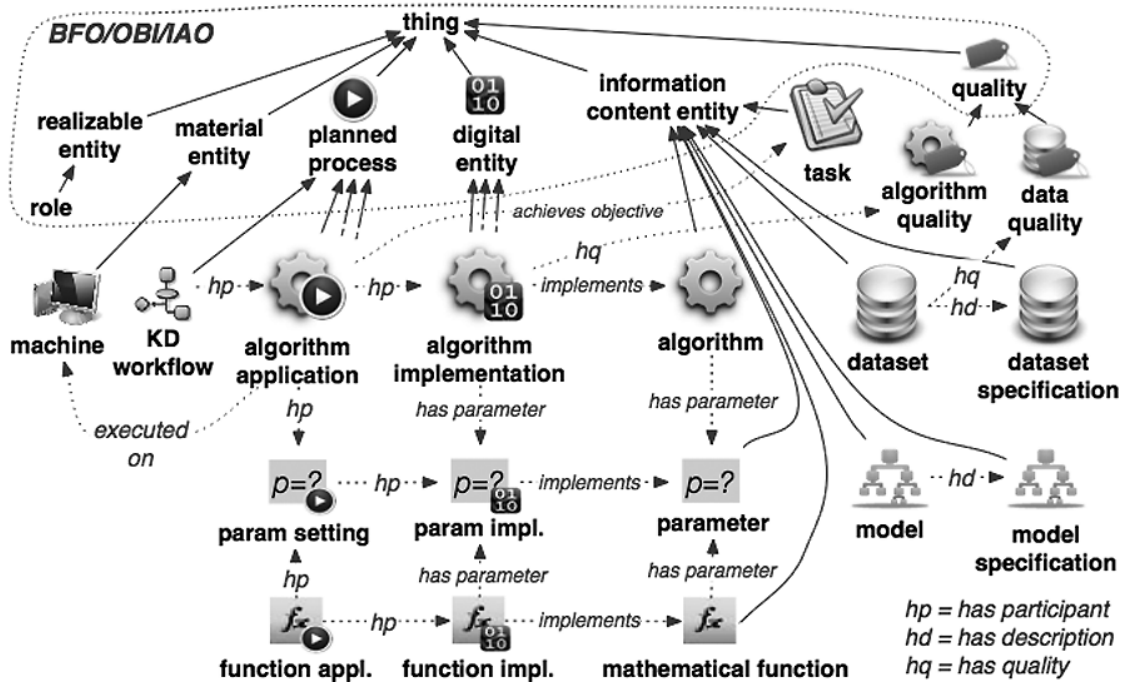

Abb. 1: Top-Level-Konzepte der Data-Mining-Ontologie.

Eine typische Datenbankabfrage zu Experimenten im Klassifikationslernen produziert große und daher leicht unübersichtliche Tabellen. Ein Plot etwa der Korrektheit nach Basis-Lernverfahren ist leicht (etwa mit Excel) erstellt, gibt aber nur unzureichenden Einblick in die reichhaltige Information dieser Datenbank. 
Daher wurde ein Plugin entworfen und implementiert, das bestehende und neue Formen zur Visualisierung hochdimensionaler Daten einsetzt (Gao, 2010).

Ein typisches Anwendungsbeispiel ist die Datenmenge „Bagging“, die 1447 Experimentalläufe verschiedener Verfahren des Bagging auf Standard-Datensets enthält. (Bagging ist eine Kombination der Anwendung eines Klassifikationslerners auf unterschiedliche Stichproben der Basisdaten, ein Ansatz, der zu wesentlich besserer Vorhersagequalität führt.) Jeder Lauf ist durch 15 Features spezifiziert, die teils die Basisdaten beschreiben, teils die Algorithmen und teils die Ergebnisse. Zwei der Features sind nominalskaliert, der Rest intervallskaliert. Mithin müssen 1447 Punkte in einem 15-dimensionalen Raum so dargestellt werden, dass auch die Effekte der verschiedenen Dimensionen inspizierbar werden.

Die erste Teilaufgabe, die sich hier stellt, ist die Suche nach einer Verteilung der 1447 Punkte über die Ebene (in der vorliegenden Arbeit kamen nur 2DVisualisierungen zur Anwendung; die Frage würde sich aber auch bei einer 3DVisualisierung fast unverändert stellen), die die Distanzen im ursprünglichen 15dimensionalen Raums möglichst wenig verzerrt, also einander nahe Punkte (ähnliche Experimentalläufe) möglichst beieinander lässt und einander ferne voneinander trennt. $\mathrm{Zu}$ diesem Zweck wurden die von acht Verfahren der Dimensionalitätsreduktion mit jeweils verschiedenen Parameterwerten produzierten 2D-Projektionen durch die Maße trustworthiness und continuity (Venna, 2007) bewertet und das Visualisierungsverfahren gewählt, das auf beiden Maßen die besten Ergebnisse lieferte. Es handelt sich um Diffusion Maps (Coifman et al., 2005) mit den Parametern $t=\sigma=1$. Die zweite Teilaufgabe ist, wie man anhand der entstandenen Basistopologie interessante Abhängigkeiten zwischen Features visualisieren kann. Zu diesem Zweck wurde jedes Feature nach [0;1] normalisiert, und für jedes Feature wurde eine eingefärbte Variante des Punkteplots erstellt, deren Farbskala von dunkelblau (0) bis rot (1) läuft. Auf kleinem Raum zeigen also 15 3D-Bilder (2 räumliche Dimensionen plus Farbe) Information, aus der Metaaussagen über alle Experimentalläufe und ihre Variablen abgeleitet werden können.

Abbildung 2 zeigt das Ergebnis. Die vom Visualisierungsverfahren gebildeten vertikalen „Stäbchen“ von Punkten entsprechen im Wesentlichen den BasisDatenmengen, wie man an den homogenen Farben der „Stäbchen“ z. B. für Feature 3 (dataset), 12 (number of missing values - diese sind natürlich jeweils gleich für verschiedene Experimentalläufe auf denselben Daten), 14 (default accuracy - Anteil der Instanzen, die zur Mehrheitsklasse gehören) oder auch 4, 5, 6, 7 sieht. Innerhalb dieser visuellen Cluster hat sich als Y-Achse eine Dimension ergeben, die v.a. der Zahl der Iterationen entspricht (1), und als X-Achse eine Dimension, die gut mit der Performanz der Landmarker-Lernverfahren (810) übereinstimmt, was sich auch in der Performanz des Gesamtverfahrens (15) widerspiegelt, ohne aus den dem Bagging zugrunde liegenden Lernverfahren (2) vorhersagbar zu sein. 


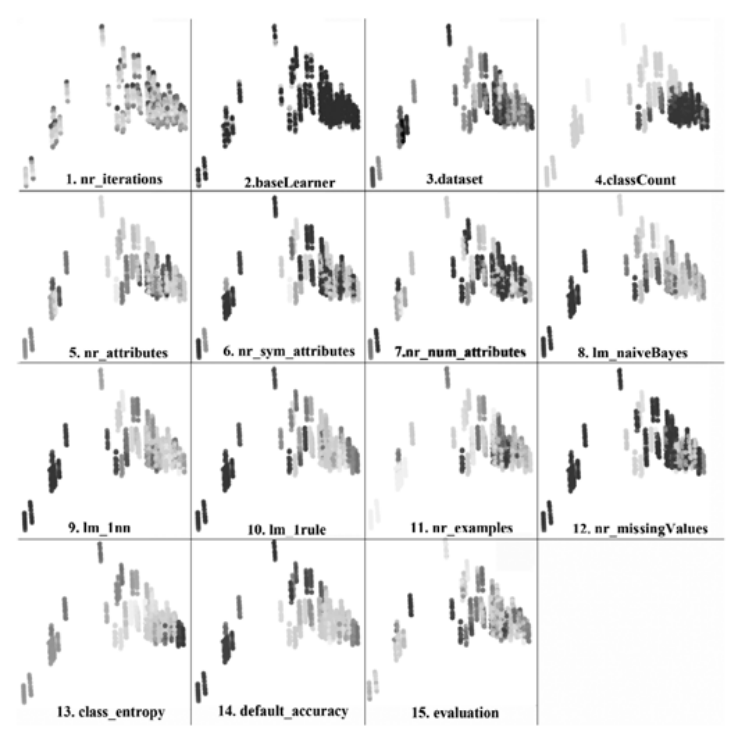

Abb. 2: Analyse der 1447 Experimentalläufe zum Bagging hinsichtlich 15 Parametern (Eine farbige Variante dieser Abbildung ist auf http://www.berendt.de/FDM.png zu finden.)

\subsubsection{Zusammenfassung}

Zukunftsorientiertes Forschungsdatenmanagement geht über die Dokumentation von Forschungsergebnissen und -prozessen hinaus und ermöglicht neue Formen der Wieder- und Weiternutzung der gespeicherten Daten. Dabei spielen Re- und Meta-Analysen dieser Daten eine besondere Rolle, und Visualisierungen als Form der explorativen Datenanalyse können wertvolle Erkenntnisse liefern und zu neuen Forschungsfragen führen. In diesem Artikel haben wir die Interoperabilität der Daten (durch gemeinsame Semantik und Syntax, realisiert durch Ontologien und Markup-Sprachen) als wichtige Voraussetzung für Analysen und den Einsatz geeigneter Verfahren zur Dimensionalitätsreduktion als Kernbestandteil geeigneter Visualisierungen identifiziert und mit einem Beispiel, einer Experimentaldatenbank zum Data Mining / maschinellen Lernen, illustriert. Die Entwicklung und Bereitstellung solcher Tools werden zu den wichtigsten Schritten der nächsten Zukunft gehören, um das Forschungsdatenmanagement offen und damit wissenschaftlich generativ zu gestalten. 


\section{Literaturhinweise}

Barbaro, M. \& Zeller, T., 2006. A face is exposed for AOL searcher no. 4417749. New York Times, 9. Aug.

Coifman, R.R. et al., 2005. Geometric diffusions as a tool for harmonic analysis and structure definition of data: Diffusion maps. Proceedings of the National Academy of Sciences, 102(21), S. 7426-7431.

Conrad, S., 1997. Föderierte Datenbanksysteme: Konzepte der Datenintegration. Berlin: Springer.

Enser, P., 2008. The evolution of visual information retrieval. Journal of Information Science, 34, S. 531-546.

Feldman, R. \& Sanger, J., 2007. The Text Mining Handbook. Advanced Approaches in Analyzing Unstructured Data. Cambridge, UK: Cambridge University Press.

Fung, B.C.M. Wang, K. Chen, R. \& Yu, P.S., 2010. Privacy-preserving data publishing: A survey on recent developments. ACM Computing Surveys, 42(4). DOI=10.1145/1749603.1749605, http://doi.acm.org/10.1145/ 1749603.1749605.

Gao, B., 2010. Advanced Visualizations of Machine Learning Behaviour. Masters Thesis. K.U. Leuven, Department of Computer Science. UDC: $681.3 *$ I20.

Hand, D.J. Smyth, P. \& Mannila, H., 2001. Principles of Data Mining. Cambridge, MA: MIT Press.

Katifori, A. et al., 2007. Ontology visualization methods - a survey. ACM Computing Surveys, 39(4). DOI=10.1145/1287620.1287621, http:// doi.acm.org/10.1145/1287620.1287621.

Nielsen, M., 2008. The future of science: Building a better collective memory. APS Physics, 17(10), 8. Online: http://www.aps.org/publications/apsnews/ 200811/upload/November-2008-Volume-17-No-10.pdf [Zugriff am 13.08.2011].

Schenk, M., Hrsg., 2010. Instandhaltung technischer Systeme. Methoden und Werkzeuge zur Gewährleistung eines sicheren und wirtschaftlichen Anlagenbetriebs. Berlin: Springer.

Schumann, H. \& Müller, M., 1999. Visualisierung: Grundlagen und allgemeine Methoden. Berlin: Springer. 
Sweeney, L., 2002. k-anonymity: A model for protecting privacy. International Journal of Uncertainty, Fuzziness and Knowledge-Based Systems, 10(5), S. 557-570.

Tukey, J.W., 1977. Exploratory Data Analysis. Reading, MA: Addison-Wesley.

University of Edinburgh Information Services, 2009. Defining Research Data. Online:http://www.ed.ac.uk/schools-departments/information-services/ services/research-support/data-library/research-data-mgmt/data-mgmt/ research-data-definition [Zugriff am 02.02.2011].

Vanschoren, J. Blockeel, H. Pfahringer, B. \& Holmes, G. (im Druck). Experiment databases. A new way to share, organize and learn from experiments. Machine Learning. Online: https://lirias.kuleuven.be/handle/ 123456789/297378 [Zugriff 13.08.2011].

Venna, J., 2007. Dimensionality Reduction for Visual Exploration of Similarity Structures. PhD Thesis. Helsinki University of Technology, Department of Computer Science and Engineering. Online: http://lib.tkk.fi/Diss/2007/ isbn9789512287529/ [Zugriff am 13.08.2011]. 